\title{
Ocular traumatology: prevention, prevention, prevention...
}

\author{
Ferenc Kuhn
}

Received: 30 December 2009 / Accepted: 30 December 2009/Published online: 28 January 2010

(C) Springer-Verlag 2010

\section{Keyword Trauma}

As one reads through the second annual trauma section of Graefe's, what primarily comes to mind is the importance of prevention.

All of us know that it is much preferable to prevent an eye injury from happening than even the most effective treatment. Should the injured eye's vision be successfully restored to normal, the patient (and the family) still underwent a scary experience, a lot of anxiety, and much inconvenience. In addition, there is substantial cost involved, direct and indirect, and lives get interrupted for extended periods of time.

Prevention has many components. At first glance, the easiest way to prevent an eye injury appears to be legislation. Once enough data have been accumulated by ophthalmologists to pinpoint a particular circumstance with a high incidence of eye injury, politicians and legislators have the basis for developing and enacting legislation that will regulate/ban the activity/circumstance in question.

A good example of such legislation is the sale and personal ("recreational") use of fireworks. There are good data available from the US showing that states allowing all or most types of fireworks have an almost ten-fold higher eye injury incidence rate than states with a "Model Law", which bans all fireworks except those used in public displays.

The article by Jing et al. in the current issue of Graefe's also emphasizes the importance of legislation. Although the

F. Kuhn $(\bowtie)$

Helen Keller Foundation,

1201 11th Ave S. Suite 300,

Birmingham, AL 35205, USA

e-mail: fkuhn@mindspring.com authors do not provide numbers, they do mention that a previously enacted ban in China resulted in the decline of the number of severe eye injuries, but the ban was then rescinded, and the number of injuries rose again. A similar experience was reported from Washington State in the US, where the easing of the restrictions lead to a $126 \%$ increase in the number of cases.

Legislation, of course, is politics, where power and money are key players. When the Eye Injury Registry of Alabama tried to have bottle rockets banned in the state, its arguments could not match the resources the retail industry was able to throw at the legislators. Although, as Jing et al. show, the risk is not limited to the operator of the fireworks (one-third of injuries occur to bystanders), it seems unrealistic to make them wear eye protection, even if the operator of the fireworks can be convinced to do so, and although only $4 \%$ of the injured eyes regained 0.5 vision, legislating fireworks use remains an uphill battle.

To a certain extent, as Kay et al. point out, the problem related to paintball ("war games") is similar. In commercial use, proper face protection has indeed been mandated, and it is easily available. Yet the fact that most injuries occur during noncommercial games shows that many people are either unaware of the danger or their attitude toward the risk is the typical one: "I know it happens, but it happens to other people, not to me." The author of this editorial recently treated a middle-aged male who lost one eye due to a paintball injury; he did not wear face protection, and he is a general surgeon.

The remedies that Kay et al. propose to reduce such injuries are sensible and have the promise to be successful, but it is questionable whether any legislative restriction on something certain people like to do in their spare time and in private would work. 
Legislation and commercial interest combined can be very powerful, as the article by Schrader and Gramer shows. Glass bottles containing carbonated drinks can explode without being mishandled; indeed, $45 \%$ of injuries in their study occurred during proper handling of the bottle. Even bottles made of supposedly thick glass (sparkling wine ["champagne"]) can spontaneously explode, and they have been found to cause a "chain reaction", as the authors report.

Such injuries are not negligible: $18 \%$ of eyes remained legally blind in the study from Germany as $27 \%$ of them had retinal injury, and $21 \%$ of the patients were under the age of 8 years. Switching to plastic bottles with screw-caps is certainly an excellent way to prevent this kind of trauma, but can we imagine a French champagne maker to serve its splendid bubbly drink in such a way?

In addition to risks that are easy or relatively easy to identify even by a lay person, there are the "weird" injuries. To have a penetrating injury from an eyelash curler is not something even an ophthalmologist would immediately identify with. While such injuries are not easy to prevent, beyond the usual advice of being careful with any object around the eyeball, the report by Ramasamy and Armstrong does raise one very important point in prevention, which leads us to the second category of prevention.

Should an injury have already occurred, the ophthalmologist (indeed, any person dealing with the injured patient) must still prevent additional trauma from making the situation even worse. Iatrogenic trauma, caused by an inappropriate intervention, is rarely talked about. It is not only more convenient to attribute any postoperative complication to the original injury, it also represents selfdefense against any legal action.

One commonly incurred, but almost never discussed, complication is retinal injury from applying "standard" cataract extraction techniques in the context of trauma. Automatically, reflectively using phacoemulsification to remove the injured lens, without any consideration for a possible posterior capsule breach with consequent vitreous prolapse is a major risk factor for iatrogenic retinal break and detachment development. The authors deserve a lot of credit for their choice of lens removal by lensectomy, rather than phacoemulsification, resisting the peer pressure to "place the IOL in the bag at all cost". Preventing secondary complications was their goal, and they were absolutely correct; no patient ever asks whether his IOL is in the bag or in the sulcus, but they will raise serious questions if they lose vision due an easily avoidable retinal complication.

The report by Plestina-Bojan et al. shows what capable and conscious anterior segment surgeons can achieve, even when faced with serious war injuries and no vitrectomy capability. Meticulous, carefully planned primary reconstruction of the anterior segment and the avoidance of primary enucleation resulted in excellent initial visual recovery while making the job of the second (vitreoretinal) surgeon much easier. Although they do not discuss this in their article, but the authors could easily have faced an additional issue in this sad age of mass casualties: how do you deal with the logistical issues associated with war injuries, how do you triage your patients?

As an ophthalmologist who had many patients referred to from the recent wars in the former Yugoslavia, the contributor of this editorial can personally testify to the sad statistics the authors mention: $33 \%$ of their patients were innocent civilians, of whom $38 \%$ were children. Unfortunately, the only, in this context sadly ironic, method of prevention that comes to mind is the infamous slogan from the sixties: Make love, not war.

This leads us to the study by Weichel et al. Again, in wartime, the military ophthalmologist's effort in preventing ocular trauma is usually restricted to offer the best available treatment to the injured soldier (or civilian, as the previous report showed). Beyond the initial damage, PVR remains the greatest risk to the injured eye, and so far no pharmaceutical prevention has proven to be effective here. Weichel et al. tested a novel surgical approach, first reported in 2004. Chorioretinectomy serves two purposes: to remove/destroy the retinal pigment epithelium, the major source of PVR in the injured eye, but also to prevent the sclera-originated fibroblasts from crawling over from the wound onto the retina, thus preventing both PVR and retinal fold formation.

As characteristic of this group of military ophthalmologists, Weichel et al. conducted a meticulous, exceptionally well done study on the effect of chorioretinectomy. Their results in preventing PVR after these high-risk injuries are impressive ( $46 \%$ of eyes developed PVR originating from the wound/impact site, as opposed to a $74 \%$ rate in the nonchorioretinectomy group, a globe survival rate of $85 \%$ vs. $45 \%$, and a final retinal reattachment rate of $62 \%$ vs. $42 \%$, respectively). Nonetheless, these results are not as good as they have been found in the ongoing international trail conducted by the International Society of Ocular Trauma (www.isotonline.org). As the authors point out, this may be due to the fact that the original protocol calls it, and for, prophylactic chorioretinectomy: prevention, rather than treatment is the goal. In the military, this early surgery (within the first 100 hours) is often impossible due to logistical issues as well as to the fact that most patients have multiorgan trauma and the eye is treated not when it is ideal but when it becomes possible.

We hope that readers will benefit from these articles and will be able to utilize the new information for the benefit of their patients, and also to prevent persons from becoming patients. Finally, the contributor of this editorial would like to thank all the authors for their contributions and Graefe's for publishing these annual trauma sections. 